\title{
Acutaxylines A and B, two novel triterpenes from Dysoxylum acutangulum
}

\begin{abstract}
Two novel triterpenes, acutaxylines A (1) and B (2) consisting of a cyclopentenone side chain at C-17 and an oxepan-2-ol, were isolated from the leaves of Dysoxylum acutangulum. The relative stereochemistry of 1 and 2 was determined by NOESY correlations. Acutaxyline B showed moderate cytotoxicity against human blood premyelocytic leukemia cells.
\end{abstract}

Keyword: Acutaxylines A; Acutaxylines B; Triterpenes; Dysoxylum acutangulum 